\title{
Interferon-lambda (IFNL) germline variations and their significance for HCC and PDAC progression: an analysis of The Cancer Genome Atlas (TCGA) data
}

Henriette Huschka and Sabine Mihm * (D)

\begin{abstract}
Background: Hepatocellular carcinoma (HCC) and pancreatic ductal adenocarcinoma (PDAC) are malignancies with a leading lethality. With reference to interferons (IFNs) known to mediate antitumor activities, this study investigated the relationship between germline genetic variations in type III IFN genes and cancer disease progression from The Cancer Genome Atlas (TCGA) data. The genetic variations under study tag a gain-or-loss-offunction dinucleotide polymorphism within the IFNL4 gene, rs368234815 [TT/AG].
\end{abstract}

Methods: The entirety of the TCGA sequencing data was used to assess genotypes of 187 patients with HCC and of 162 patients with PDAC matched for ethnicity. Stratified for IFNL genotypes, both cohorts were subjected to time-to-event analyses according to Kaplan-Meier with regard to the length of the specific progression free interval (PFI) and the overall survival (OS) time as two clinical endpoints for disease progression.

Results: Logrank analysis revealed a significant relationship between IFNL genotypes and disease outcome for PDAC. This relationship was not found for HCC. A multiple Cox regression analysis employing patients' age, tumor grade and tumor stage as further covariates proved IFNL genotypes to be independent predictors for PDAC disease outcome.

Conclusion: This repository-based approach unveiled clinical evidence suggestive for an impact of IFNL germline variations for PDAC progression with an IFNL haplotype predisposing for IFNL4 expression being favorable.

Keywords: Interferon-lambda4 (IFNL4), IFNL4 rs368234815, Type III interferons, Hepatocellular carcinoma (HCC), Pancreatic ductal adenocarcinoma (PDAC), Antitumor host response, Progression free interval (PFI), Overall survival (OS)

\section{Background}

Hepatocellular carcinoma (HCC) and pancreatic ductal adenocarcinoma (PDAC) are leading causes of cancerrelated deaths [1]; they belong to the most lethal malignancies with 5 -yr survival rates $<20 \%[2,3]$. According to Hanahan and Weinberg, dysregulated cellular

\footnotetext{
* Correspondence: smihm@med.uni-goettingen.de

* Correspondence: smihm@med.uni-goettingen.de
Department of Gastroenterology, Gastrointestinal Oncology, and Endocrinology, University Medical Center Goettingen, Robert-Koch-Str. 40, 37075 Goettingen, Germany
}

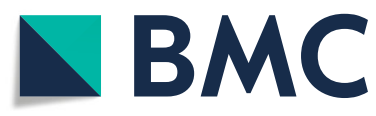

(c) The Author(s). 2020 Open Access This article is licensed under a Creative Commons Attribution 4.0 International License, which permits use, sharing, adaptation, distribution and reproduction in any medium or format, as long as you give appropriate credit to the original author(s) and the source, provide a link to the Creative Commons licence, and indicate if changes were made. The images or other third party material in this article are included in the article's Creative Commons licence, unless indicated otherwise in a credit line to the material. If material is not included in the article's Creative Commons licence and your intended use is not permitted by statutory regulation or exceeds the permitted use, you will need to obtain permission directly from the copyright holder. To view a copy of this licence, visit http://creativecommons.org/licenses/by/4.0/. The Creative Commons Public Domain Dedication waiver (http://creativecommons.org/publicdomain/zero/1.0/) applies to the data made available in this article, unless otherwise stated in a credit line to the data.

pathways that transform growth of normal cells into neoplasms are regarded as 'hallmarks of cancer' [4]. The evasion of the host's immune defense, later on, has been recognized as one further principle of promoting malignant growth [4]. The impact of the host's immune system for disease progression is underscored by the recent successful clinical translation of immunotherapeutic strategies [5].

Understanding that not all patients benefit from cancer immunotherapy, the term 'cancer immune responsiveness' 
(CIR) has been coined [6]. Germline genetic variants have been proposed to contribute to CIR including those in IFN signaling genes [6]. IFNs and their effectors have been known for long not only to mediate antiviral but also to edit antitumor host responses [7, 8]. They divide into type I $\left(\right.$ IFN- $\left.\alpha_{n} / \beta\right)$, type II $($ IFN- $\gamma$ ) and type III (IFN- $\left.\lambda_{1-4}\right)$. Among the genes encoding IFN species, only one, type III IFNL4, harbors a common exonic gain-or-loss-of-function variation: while the phylogenetically older variant $\Delta G$ enables functional IFN- $\lambda_{4}$ protein expression, the knockout variant TT causes a frameshift thereby disrupting the open reading frame and preventing translation $[9,10]$. This germline dinucleotide polymorphism, IFNL4 rs368234815 $[\mathrm{TT} / \Delta \mathrm{G}]$ (merged into IFNL4 rs11322783) thus reflects the ongoing process of pseudogenization dividing human beings into those who are predisposed to express IFN- $\lambda_{4}$ protein and into those who are not [11]; it associates with clearance of hepatitis $\mathrm{C}$ virus $(\mathrm{HCV})$ and a variety of other disease conditions (reviewed in [12]).

In the context of viral infections, generally, an IFN$\lambda_{4}$ creating genetic background rather was found to be unfavorable for the host. This counter-intuitive relationship was first recognized for $\mathrm{HCV}$ infection, when the IFN- $\lambda_{4}$ creating genotypes were shown to be in LD with those IFNL variants that had been identified before to be correlated with poor clearance of $\mathrm{HCV}$ infection in genome wide association studies (GWAS) on main ethnic populations [10, 13]. Similarly, in human immunodeficiency virus (HIV) infection, the IFN- $\lambda_{4}$ creating genotype was found to be associated with a higher prevalence of AIDS [14] while the non-encoding genotype associates with a lower probability to acquire HIV $[15,16]$. Also cytomegalovirus (CMV) reactivation is described to be more prevalent in patients encoding for a functional IFN- $\lambda_{4}$ protein $[17,18]$.

The availability of collaborative comprehensive data repositories enables analyses of patient material on a whole genome scale and on large sample sizes. The Cancer Genome Atlas (TCGA) database provides datasets on more than 11,000 cancer patients across 33 tumor entities to the scientific community. Besides demographic and clinical data, TCGA comprises whole exome DNA and RNA sequencing data not only of tissue samples derived from primary tumors but also from corresponding non-malignant material, the latter giving rise to patients' germline genetic background.

By employing TCGA datasets, this study aimed at finding clinical evidence for or against an impact of IFNL germline variations for $\mathrm{HCC}$ or PDAC progression. Using the Kaplan-Meier estimator, disease progression was assessed by (i) the length of the specific progression-free interval (PFI) and by (ii) the overall survival (OS) time as two clinical outcome endpoints. A multivariate Cox proportional-hazards model was applied considering patients' age, tumor grade, and tumor stage as covariates along with IFNL genotypes.

\section{Methods \\ TCGA data portal}

Analyses are based upon data generated by TCGA (phs000178.v10.p8) which is managed by the NCI and NHGRI. Specifically, projects on HCC (TCGA-LIHC; https://portal.gdc.cancer.gov/projects/TCGA-LIHC) and on PDAC (TCGA-PAAD; https://portal.gdc.cancer.gov/ projects/TCGA-PAAD) were included. The access to controlled datasets was approved by $\mathrm{NIH}$ (project ID 20041). Open access demographic (gender, age at diagnosis, race and ethnicity) and clinical data (tumor grade and stage, specific PFI, OS time) were gathered from a curated and standardized dataset named TCGA PanCancer Clinical Data Resource (TCGA-CDR) with a focus on clinical outcome endpoints [19].

\section{Reading-out IFNL genotypes}

Controlled access whole exome sequencing (WXS) reads of non-malignant tissue (code $11 \mathrm{~A}$ ) or peripheral blood leucocyte (code 10A) DNA, or sequencing reads of non-malignant tissue RNA, were cut down to the region spanning the IFNL gene cluster (chr19: 39, $230,000-39,300,000$ ) by using the BAM slice tool before downloading. By using the NCBI genome workbench software, the truncated sequence files were aligned to chromosome 19 of the human genome reference assembly GRCh38.p12. Depending on the depth of coverage, genotypes of up to five nucleotide polymorphisms were read out (Fig. 1). According to established criteria, a coverage of 20-30 sequence reads was considered reasonably confident. For heterozygous calls, both alleles should have an allele-call score $>10$ and the ratio of their scores should be $<3$. Call rates for both, the HCC and the PDAC cohort under study, reached $100 \%$. Identical genotypes were obtained irrespective of whether malignant or nonmalignant material was analyzed, on a sample basis.

\section{Statistical analysis}

Exact test for deviation from Hardy-Weinberg equilibrium (HWE) was performed using an online calculator (http://www.dr-petrek.eu/documents/HWE.xls). Linkage disequilibrium (LD) coefficients $\mathrm{D}^{\prime}$ and $\mathrm{r}^{2}$ were enquired from the Web-based application LDlink (https://ldlink. nci.nih.gov) which refers to data of the 1000 Genomes Project (Phase 3, Version 5), by using the LDmatrix tool.

All other statistical analyses including time-to-event analysis according to the Kaplan-Meier method, and 


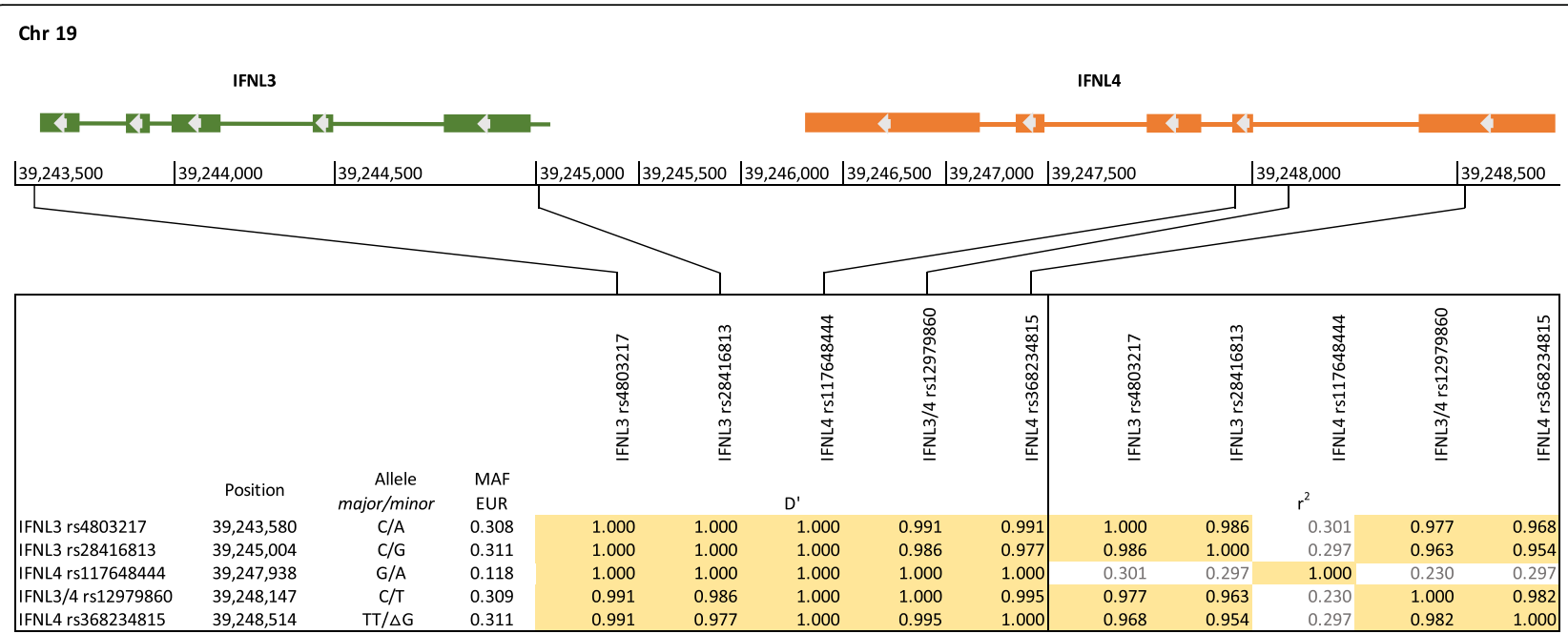

Fig. 1 Localization of IFNL gene polymorphisms under study and calculation of LD. The 5 IFNL gene polymorphisms of interest are located within the $5^{\prime}$ and $3^{\prime}$ termini of the IFNL3 gene and within exon 1 and 2 and intron 1 of IFNL4 gene on chromosome 19 as shown. The normalized coefficients of $L D\left(D^{\prime}\right)$ and the correlation coefficients $\left(r^{2}\right)$ were calculated on data provided by the 1000 Genomes Project for individuals of European ancestry $(n=503)$. With the exception of IFNL4 rs 117648444 , they qualify as mutual tagSNPs

uni- and multivariate Cox proportional-hazard models were performed in $\mathrm{R}$ version 3.5.2 [20]. $P$-values $\leq 0.05$ were considered statistically significant.

\section{Results}

\section{The TCGA sample}

The TCGA database contains data on 377 patients with hepatocellular carcinoma (HCC, C22.0) and on 185 patients with pancreatic ductal adenocarcinoma (PDAC, C25.-). They are assigned as African American, Asian, Native American, White, or of unknown ancestry (in numbers: 17 (4.5\%) / $161(42.7 \%)$ / $2(0.5 \%)$ / 187 $(49.6 \%) / 10(2.7 \%)$ and $7(3.8 \%) / 11(5.9 \%) / 0(0 \%) /$ $162(87.6 \%) / 5$ (2.7\%), respectively). Besides demographical parameters, TCGA provides comprehensive clinical documentation. For instance, potential risk factors for the development of $\mathrm{HCC}$ are specified, as are infections with hepatitis viruses (e.g. HBV, HCV), intoxications (e.g. ethanol consumption), metabolic conditions (e.g. fatty liver disease), or others, or combinations of those. A descriptive, closer view on the data expectedly revealed $\mathrm{HBV}$ infection and $\mathrm{HBV} / \mathrm{HCV}$ co-infections to be more prevalent among Asian patients, while isolated $\mathrm{HCV}$ infections and non-viral etiologies predominate among white patients (data not shown). Furthermore, patients with $\mathrm{HBV}$ infections featured more advanced tumor grades, and patients with $\mathrm{HBV} / \mathrm{HCV}$ coinfections presented higher tumor stages (data not shown). To avoid bias due to ethnicity-related environmental differences, these observations prompted us to confine to the largest possible homogenous sample, i.e. the TCGA white patients, which were $n=187$ for HCC and $n=162$ for PDAC.
Regarding etiology, HCC patients divide into those with no or unknown history of primary risk factors $(n=$ $54)$, with non-viral risk factors $(n=43)$, with confirmed HBV $(n=42)$ or HCV $(n=31)$ infection or with HBV/ HCV co-infections $(n=41)$ (data not shown). Most of them - aged 63.3 yrs. on average at diagnosis - presented with tumor grade G2 (55.7\%) and tumor stage I (47.3\%) (Tab. 1). Starting at the time of diagnosis, the length of the median observation period for disease progression in terms of the specific PFI was 12.0 mo. During this period, 99 patients faced an event while 88 were censored. Regarding OS time, the median observation period was $22.1 \mathrm{mo}$. During this period, 77 patients deceased while 110 being censored. Median specific PFI amounts up to $19.7 \mathrm{mo}$, median OS time was 45.9 mo (Tab. 1).

PDAC patients aged $65.4 \mathrm{yrs}$. on average at the time of diagnosis presented above all with tumor grade G2, too, but compared to the HCC cohort, with a more advanced tumor stage in the majority of cases. Details to median observation periods and events are listed in Table 1. The median OS time was $20.2 \mathrm{mo}$, half the less of that for the HCC cohort.

\section{Genotyping}

Patients' genotypes were raised from up to five polymorphic sites within the IFNL gene cluster by aligning whole exome DNA and RNA sequence reads from nonmalignant material to a reference genome. Coverage at IFNL4 rs368234815 was found to be insufficient for most of the HCC and PDAC samples. An LD analysis based on the data of the 1000 Genomes Project and adjusted for the European population demonstrates that all polymorphic 
Table 1 Patient characteristics

\begin{tabular}{|c|c|c|}
\hline & $\begin{array}{l}\text { HCC } \\
(n=187)\end{array}$ & $\begin{array}{l}\text { PDAC } \\
(n=162)\end{array}$ \\
\hline Age, mean \pm SD [years] & $63.3 \pm 13.8$ & $65.4 \pm 10.8$ \\
\hline Gender, m/f [n] & $105 / 82$ & $92 / 70$ \\
\hline \multicolumn{3}{|l|}{${ }^{1}$ Tumor grade [n (\%)] } \\
\hline G1 & $34(18.6)$ & $28(17.4)$ \\
\hline G2 & $102(55.7)$ & $84(52.2)$ \\
\hline G3 & $46(25.1)$ & $47(29.2)$ \\
\hline G4 & $1(0.5)$ & $2(1.2)$ \\
\hline \multicolumn{3}{|l|}{${ }^{2}$ Tumor stage $[\mathrm{n}(\%)]$} \\
\hline । & $80(47.3)$ & $20(12.6)$ \\
\hline$\|$ & $44(26.0)$ & $130(81.8)$ \\
\hline III & $41(24.3)$ & $4(2.5)$ \\
\hline IV & $4(2.4)$ & $5(3.1)$ \\
\hline \multicolumn{3}{|l|}{ Specific PFI } \\
\hline median observation period (mo) & 12.0 & 12.1 \\
\hline event / censored [n(\%)] & 99 (52.9) / 88 (47.1) & $93(57.4) / 69$ (42.6) \\
\hline median specific PFI (mo) & 19.7 & 16.2 \\
\hline \multicolumn{3}{|l|}{ OS time } \\
\hline median observation period (mo) & 22.1 & 15.3 \\
\hline event / censored [n(\%)] & $77(41.2) / 110(58,8)$ & 86 (53.1) / 76 (46.9) \\
\hline median OS (mo) & 45.9 & 20.2 \\
\hline \multicolumn{3}{|l|}{ IFNL3 genotypes } \\
\hline rs4803217 CC:CA:AA [n(\%)] & 79 (42.2): 89 (47.6): 19 (10.2) & - \\
\hline rs28416813 CC:CG:GG [n(\%)] & - & 76 (46.9): 69 (42.6): 17 (10.5) \\
\hline MAF & 0.340 & 0.318 \\
\hline HWE & $p=0.40$ & $p=0.82$ \\
\hline
\end{tabular}

${ }^{1}$ Data on tumor grade were available for 183 and 161 patients with HCC and PDAC, respectively

${ }^{2}$ Data on tumor stage were available for 169 and 159 patients with HCC and PDAC, respectively

sites under study are in a nearly complete LD to each other (Fig. 1). Due to similar minor allele frequencies (MAF), four of them qualify as mutual tagSNPs (Fig. 1). Based on sequencing coverage rates, IFNL3 rs4803217 and IFNL3 rs28416813 were chosen as surrogates for the gainor-loss dinucleotide polymorphism IFNL4 rs368234815 for HCC and PDAC patients, respectively.

For HCC patients, genotype distribution of the surrogate SNP IFNL3 rs4803217 was 79 (42.2\%): 89 (47.6\%): 19 (10.2\%) (CC:CA:AA), meeting HWE. With a MAF of 0.340 the A allele carriers $(n=108)$ are supposed to correspond to those encoding a functional IFN- $\lambda_{4}$ protein, while the $\mathrm{C}$ allele homozygotes resemble the IFNL4 knockouts (Tab. 1).

Surrogate IFNL3 rs28416813 genotype distribution of PDAC patients was 76 (46.9\%): 69 (42.6\%): 17 (10.5\%) (CC: CG:GG) and was also found to match HWE. With a MAF of $0.318,86$ patients who are $G$ allele carriers are supposed to be capable of expressing IFN- $\lambda_{4}$ protein (Tab. 1).

\section{Analysis of disease progression with regard to IFNL} genotypes

The length of the specific PFI and the OS time were chosen as clinical endpoints for disease progression. By employing Kaplan-Meier analyses, both parameters were analyzed with regard to patients' IFNL genotypes.

For HCC patients, the length of the median specific PFI did not relate to the number of the IFNL3 rs4803217 alleles (gene dosage) (i.e., $18.4 \mathrm{mo}: 21.0 \mathrm{mo}$ : $14.9 \mathrm{mo}$ for CC: CA: AA). The lack of this relationship became apparent also in Kaplan-Meier graphs (Fig. 2). The logrank test confirmed a lack of a significant difference in the length of the specific PFI between IFNL3 rs4803217 C allele homozygotes and A allele carriers $(p=0.65)$. Similar nonsignificant results were obtained when the OS time as an endpoint of disease progression was analyzed with regard to IFNL3 genotypes ( $p=0.87$, logrank test).

For PDAC patients, the length of the median specific PFI was shorter for IFNL3 rs28416813 CC homozygotes 
than for $\mathrm{G}$ allele carriers (i.e., 12.8 mo: 19.5 mo: 17.9 mo for CC: CG: GG). This relationship manifests in the time-to-event Kaplan-Meier graphs (Fig. 2). The logrank test revealed a significant difference of the length of the specific PFI with CC homozygotes (corresponding to patients not encoding IFNL4) showing earlier disease progression than $\mathrm{G}$ allele carriers $(p=0.01)$. Similar results were obtained for OS time as a further clinical endpoint $(p=0.05$, logrank test $)$.

\section{Uni- and multivariate analyses of disease progression}

In order to find out whether IFNL genotype is an independent parameter relating to disease progression, a multivariate Cox proportional-hazard model was applied. Parameters that revealed significant association in the univariate analysis were considered as covariates. These are patients' age, tumor grade and tumor stage for HCC patients, and patients' age, tumor grade, tumor stage and IFNL genotypes for PDAC patients, respectively.

For HCC, univariate analysis revealed tumor grade and tumor stage to be significantly related to the length of the specific PFI, while patients' age was found to be significantly related to OS time (Tab. 2). Multivariate analysis revealed lower tumor grade compared to higher grade (G1 vs G2) tend to be independently associated with a twice as long specific PFI. A higher stage was found to be related to an up to 2-fold shortened specific PFI. Regarding OS time, multivariate analysis revealed patients' age at diagnosis to be the only independent predictor (Tab. 2).

For PDAC, univariate analyses similarly revealed tumor grade and tumor stage to be related to the length of the specific PFI, in addition to IFNL genotypes (Tab. 3). Multiple analysis proved tumor stage and IFNL genotypes to be independently and significantly related to disease

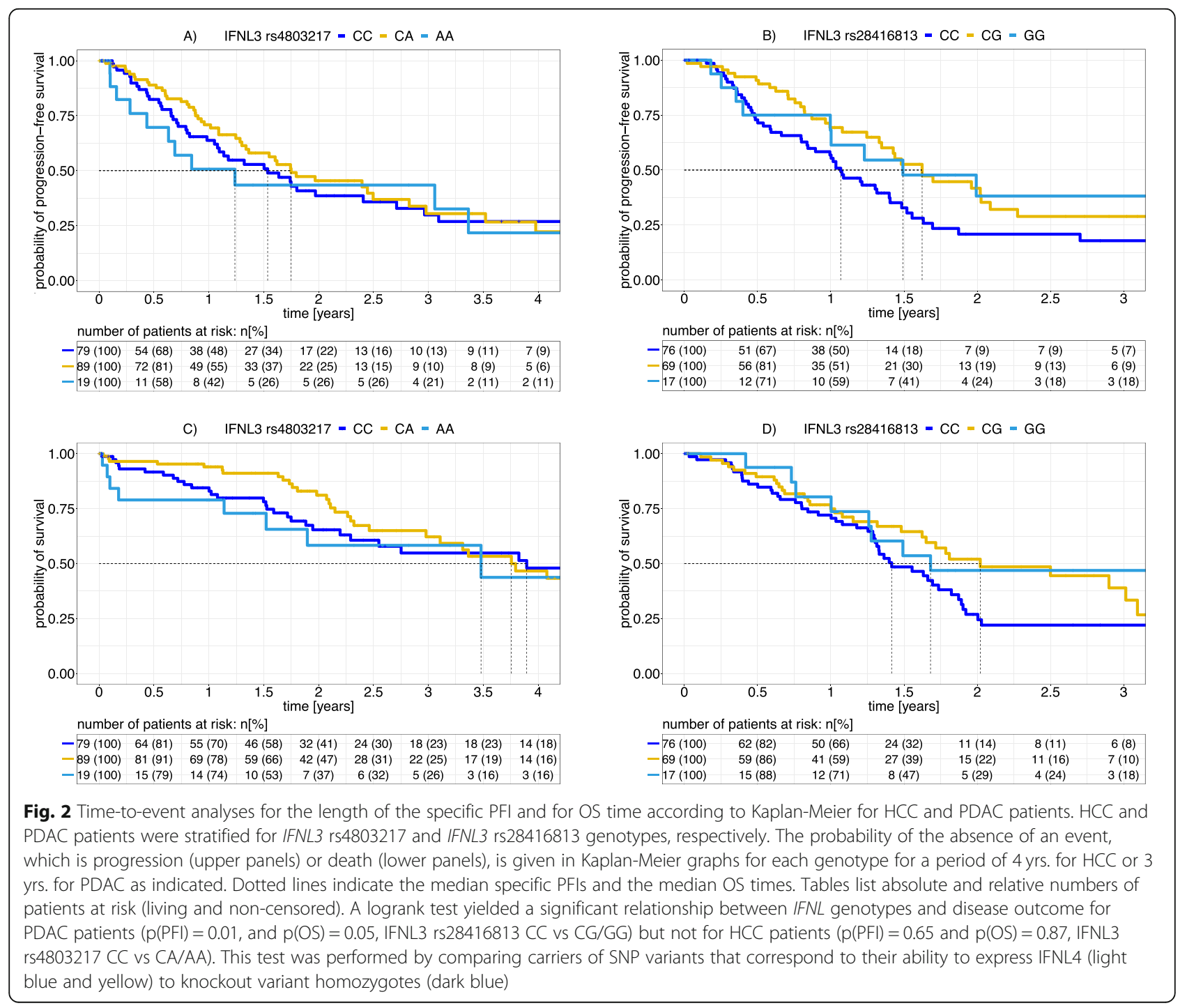


progression in terms of the length of the specific PFI. Patients with tumor stage I face a $63 \%$ less probability for progression when compared to patients with stage II ( $p=$ $0.03)$. Patients with $I F N L$ genotypes corresponding to the ability to express a functional IFN- $\lambda_{4}$ protein had a $39 \%$ lower risk to face progression than patients with an IFNL4 knockout haplotype ( $p=0.02)$ (Tab. 3).

Regarding OS time, patients' age was found to be significantly related to this endpoint, too, in the univariate model. The multivariate model revealed patients with tumor stage II to face higher risk of mortality than patients with tumor stage I $(p=0.06)$, however, with reservations (Tab. 2). Multivariate analysis, moreover, yielded a tendency of an association for IFN $-\lambda_{4}$ creating genotypes and a lower risk to decease (32\%) when compared to IFNL4 knockout haplotypes $(p=0.09)$.

\section{Discussion}

Based on TCGA datasets, this study revealed significant associations between IFNL germline variations and progression of PDAC in terms of the length of the specific PFI and the OS time as two clinical endpoints. These IFNL variations are in nearly complete LD to a dinucleotide polymorphism that controls IFNL4 gene expression (IFNL4 rs368234815). By performing a multiple regression analysis including patients' age at diagnosis, tumor stage, and tumor grade as further covariates, IFNL variation was proven to be an independent parameter for the length of the specific PFI $(p=0.02)$ and with a tendency to significance also for OS time $(p=0.09)$. This relationship was not observed for a cohort of patients matched for ethnicity but diagnosed for HCC.

A genetic background corresponding to the ability to express a functional IFN $-\lambda_{4}$ protein, i.e. carriers of the IFNL4 rs368234815 creating $\Delta \mathrm{G}$ allele, was found to be related to a delayed progression of PDAC disease, i.e. being favorable. As outlined above, in the context of viral diseases, an IFN $-\lambda_{4}$ creating genetic background - in general - is unfavorable for the host.

This disadvantage is also seen in the context of some cancer diseases, in particular for cancer entities with a virus related etiology. For instance, the IFNL4 rs368234815 $\Delta \mathrm{G}$ allele was shown to be associated with prostate cancer among men at increased risk of sexually transmitted infections [21]. In an independent study, this allele was shown to be related to significant decreased overall survival of African-American men with prostate cancer [22]. Moreover, susceptibility to AIDSrelated Kaposi's sarcoma was also found to be associated with genotypes predicted to produce an active IFN- $\lambda_{4}[23]$.

In contrast, for PDAC, an entity for which no virus-related etiology is supposed, we found the genetic predisposition to encode for IFN- $\lambda_{4}$ to be favorable for the outcome in terms of the length of the

Table 2 Uni- and multivariate Cox proportional-hazard analyses for disease progression in HCC patients

\begin{tabular}{|c|c|c|c|c|c|}
\hline \multirow[t]{2}{*}{ Disease progression criteria } & \multicolumn{2}{|c|}{ Univariate analysis } & \multicolumn{2}{|c|}{ Multivariate analysis } & \multirow{2}{*}{$\begin{array}{l}\text { Schoenfeld residuals } \\
p \text {-value }\end{array}$} \\
\hline & HR (95\% Cl) & $\overline{p \text {-value }}$ & HR $(95 \% \mathrm{Cl})$ & $\overline{p \text {-value }}$ & \\
\hline \multicolumn{6}{|l|}{${ }^{a}$ Length of the specific PFI } \\
\hline Age & $0.99(0.98-1.00)$ & 0.15 & - & - & - \\
\hline Gender (m/f) & $0.87(0.58-1.29)$ & 0.48 & - & - & - \\
\hline Tumor grade (G1 vs. G2) & $0.54(0.30-0.97)$ & $0.04^{*}$ & $0.54(0.28-1.07)$ & $0.08\left(^{*}\right)$ & 0.14 \\
\hline Tumor grade (G3/G4 vs. G2) & $1.14(0.73-1.80)$ & 0.56 & $1.25(0.78-2.02)$ & 0.35 & \\
\hline Tumor stage (stage II vs. stage I) & $1.78(1.06-3.00)$ & $0.03^{*}$ & $1.73(1.01-2.93)$ & $0.04^{*}$ & 0.02 \\
\hline Tumor stage (stage III/IV vs. stage I) & $1.96(1.19-3.24)$ & $0.01^{*}$ & $2.12(1.26-3.55)$ & $<0.01^{*}$ & \\
\hline IFNL3 rs4803217 genotype (CA/AA vs. CC) & $1.10(0.74-1.63)$ & 0.65 & - & - & - \\
\hline \multicolumn{6}{|l|}{${ }^{\mathrm{a}}$ OS time } \\
\hline Age & $1.03(1.01-1.05)$ & $0.02^{*}$ & $1.02(1.00-1.04)$ & $0.02^{*}$ & 0.93 \\
\hline Gender (m/f) & $1.10(0.70-1.73)$ & 0.67 & - & - & - \\
\hline Tumor grade (G1 vs. G2) & $0.50(0.25-1.00)$ & $0.05^{*}$ & $0.53(0.27-1.07)$ & $0.08\left(^{*}\right)$ & 0.25 \\
\hline Tumor grade (G3/G4 vs. G2) & $0.91(0.53-1.55)$ & 0.72 & $1.01(0.58-1.75)$ & 0.96 & \\
\hline Tumor stage (stage II vs. stage I) & $0.94(0.50-1.79)$ & 0.85 & - & - & - \\
\hline Tumor stage (stage III/IV vs. stage I) & $1.31(0.75-2.26)$ & 0.34 & - & - & - \\
\hline IFNL3 rs4803217 genotype (CA/AA vs. CC) & $1.11(0.71-0.74)$ & 0.66 & - & - & - \\
\hline
\end{tabular}

${ }^{a}$ Cox regression analyses were performed for complete data sets $(n=167)$

* significant $p$-value $(p \leq 0.5)$

(*) tendency to significant $p$-value $(p \leq 0.1)$

Results with a $95 \% \mathrm{Cl}$ including 1 and/or a significant Schoenfeld residual have to be considered with reservation. 
Table 3 Uni- and multivariate Cox proportional-hazard analyses for disease progression in PDAC patients

\begin{tabular}{|c|c|c|c|c|c|}
\hline \multirow[t]{2}{*}{ Disease progression criteria } & \multicolumn{2}{|c|}{ Univariate analysis } & \multicolumn{2}{|c|}{ Multivariate analysis } & \multirow{2}{*}{$\begin{array}{l}\text { Schoenfeld residuals } \\
p \text {-value }\end{array}$} \\
\hline & HR (95\% CI) & $p$-value & HR (95\% CI) & $p$-value & \\
\hline \multicolumn{6}{|l|}{ Lenghth of the specific PFI } \\
\hline Age & $1.01(0.99-1.03)$ & 0.27 & - & - & - \\
\hline Gender (m/f) & $1.13(0.75-1.70)$ & 0.56 & - & - & - \\
\hline Tumor grade (G1 vs. G2) & $0.63(0.33-1.20)$ & 0.16 & $0.99(0.51-1.90)$ & 0.97 & 0.24 \\
\hline Tumor grade (G3/G4 vs. G2) & $1.57(1.01-2.45)$ & $0.05^{*}$ & $1.34(0.85-2.12)$ & 0.20 & \\
\hline Tumor stage (stage I vs. stage II) & $0.32(0.14-0.74)$ & $0.01^{*}$ & $0.37(0.15-0.89)$ & $0.03^{*}$ & 0.05 \\
\hline Tumor stage (stage III/ IV vs. stage II) & $0.99(0.46-2.16)$ & 0.98 & $1.09(0.50-2.39)$ & 0.83 & \\
\hline IFNL3 rs28416813 genotype (CG/GG vs. CC) & $0.60(0.40-0.91)$ & $0.02^{*}$ & $0.61(0.40-0.93)$ & $0.02^{*}$ & 0.60 \\
\hline \multicolumn{6}{|l|}{${ }^{\mathrm{a}}$ OS time } \\
\hline Age & $1.03(1.01-1.05)$ & $0.01^{*}$ & $1.01(1.00-1.04)$ & 0.13 & 0.67 \\
\hline Gender (m/f) & $1.26(0.83-1.93)$ & 0.28 & - & - & - \\
\hline Tumor grade (G1 vs. G2) & $0.76(0.24-1.00)$ & $0.05^{*}$ & $0.76(0.36-1.60)$ & 0.47 & 0.24 \\
\hline Tumor grade (G3/G4 vs. G2) & $1.35(0.85-2.13)$ & 0.20 & $1.13(0.70-1.81)$ & 0.61 & \\
\hline Tumor stage (stage I vs. stage II) & $0.37(0.16-0.86)$ & $0.02^{*}$ & $0.40(0.16-1.04)$ & $0.06\left(^{*}\right)$ & 0.01 \\
\hline Tumor stage (stage III/ IV vs. stage II) & $0.90(0.36-2.23)$ & 0.82 & $0.95(0.38-2.40)$ & 0.92 & \\
\hline IFNL3 rs28416813 genotype (CG/GG vs. CC) & $0.66(0.43-1.00)$ & $0.05^{*}$ & $0.68(0.44-1.07)$ & $0.09(*)$ & 0.66 \\
\hline
\end{tabular}

${ }^{a}$ Cox regression analyses were performed for complete data sets $(n=158)$

* significant $\mathrm{p}$-value $(p \leq 0.5)$

$\left(^{*}\right)$ tendency to significant $p$-value $(p \leq 0.1)$

Results with a $95 \% \mathrm{Cl}$ including 1 and/or a significant Schoenfeld residual have to be considered with reservation.

specific PFI and of the OS time. Even if biology of IFN- $\lambda$ species is not yet completely understood, this is in accordance with the supposed general antitumor activity of type III IFNs [24, 25].

TCGA also provides information on cancer treatments. Where available, data comprise the type of the therapy, its starting date and duration, and the response to it. All of the HCC patients included into our analysis are documented to have received surgery, i.e. liver lobectomy or segmentectomy. Some of them received ablation $(n=40)$, adjuvant radiation $(n=7)$, or one or several regimens of chemotherapy $(n=12)$. Likewise, all of the PDAC cases under investigation were subjected to partial or total pancreatectomy. Some of them received adjuvant radiation therapy $(n=37)$ or adjuvant chemotherapy $(n=109)$. Our analyses were performed disregarding therapeutic schemes or their outcomes, which is a limitation. However, our analyses focusing on disease outcome in terms of the length of the specific PFI and OS time, are based on the assumption that patients were receiving the best possible care according to their individual health conditions. Nevertheless, the significant but weak association between IFNL genotype and clinical outcome for PDAC patients in the whole cohort might mask stronger associations within subgroups, e.g. among patients who are responding or nonresponding to a cytostatic therapy. Data thus might reflect therapy responsiveness that, in turn, might translate into disease outcome. Accordingly, this association between IFNL variants and disease progression might be more prominent for PDAC than for HCC patients due to a higher proportion of patients subjected to chemotherapy, i.e. $109 / 162$ (73.3\%) vs $12 / 187$ (6.4\%), respectively. Whether treatment response is underlying the association between IFNL genotypes and cancer disease progression needs to be addressed in a separate analysis with a higher sample number. Alternatively, the lack of a relationship between IFNL genotypes and HCC progression might be related to that proportion of cases with viral etiology that distinguishes the HCC cohort from the PDAC cohort. The HCC cohort under study includes more than half of the patients $(61 \%)$ with HBV infection $(n=42)$, with HCV infection $(n=31)$ or with $\mathrm{HBV} / \mathrm{HCV}$ coinfections $(n=41)$.

\section{Conclusion}

By employing a collaborative oncologic data repository with a given number of cases, TCGA facilitated the exploratory mining of clinical evidence suggestive for of an impact of IFNL germline variations on PDAC cancer progression. An IFNL haplotype predisposing for IFNL4 gene expression appeared to be favorable for the host, which is in line with the concept of antitumor activities of type III IFNs. Further analyses will regard therapeutic interventions as additional variates. 


\section{Abbreviations}

CIR: Cancer immune responsiveness; CMV: Cytomegalovirus; HBV: Hepatitis B virus; HCV: Hepatitis C virus; HCC: Hepatocellular carcinoma; HIV: Human immunodeficiency virus; HWE: Hardy-Weinberg equilibrium; IFN: Interferon; IFNL4: Interferon-lambda 4 gene; LD: linkage disequilibrium; MAF: Minor allele frequency; OS: Overall survival; PDAC: Pancreatic ductal adenocarcinoma; PFI: Progression free interval; SNP: Single nucleotide polymorphism; TCGA: The Cancer Genome Atlas

\section{Acknowledgements}

The results published here are based in whole upon data generated by The Cancer Genome Atlas (TCGA, phs000178.v10.p8) managed by the $\mathrm{NCl}$ and NHGRI. Information about TCGA can be found at https://www.cancer.gov/tcga. The authors would like to thank Dr. Andreas Leha, Department of Medical Statistics, for assisting with the modelling of the criteria for multivariate analyses.

This publication is financially supported by the open access publication fund of the University of Goettingen.

\section{Authors' contributions}

The study was designed by $\mathrm{HH}$ and $\mathrm{SM}$, data analysis and interpretation was performed by $\mathrm{HH}$ and SM, statistical analyses were conducted by $\mathrm{HH}$, the manuscript was prepared by SM, and all authors have read and approved the manuscript.

\section{Funding}

Open Access funding enabled and organized by Projekt DEAL.

\section{Availability of data and materials}

The raw datasets supporting the conclusions of this article are available via The Cancer Genome Atlas (TCGA) web portal (https://portal.gdc.cancer.gov/ projects/TCGA-LIHC and https://portal.gdc.cancer.gov/ projects/TCGA-PAAD for HCC and PDAC datasets, respectively).

\section{Ethics approval and consent to participate}

The usage of TCGA datasets is covered by specific TCGA ethics policies including a compulsory Data Use Certification Agreement.

\section{Consent for publication}

Not applicable.

\section{Competing interests}

The authors declare that they have no competing interests.

Received: 10 August 2020 Accepted: 29 October 2020

Published online: 23 November 2020

\section{References}

1. Global Burden of Disease Cancer C, Fitzmaurice C, Akinyemiju TF, Al Lami FH, Alam T, Alizadeh-Navaei R, et al. Global, Regional, and National Cancer Incidence, Mortality, Years of Life Lost, Years Lived With Disability, and Disability-Adjusted Life-Years for 29 Cancer Groups, 1990 to 2016: A Systematic Analysis for the Global Burden of Disease Study. JAMA Oncol. 2018:4(11):1553-68.

2. Bray F, Ferlay J, Soerjomataram I, Siegel RL, Torre LA, Jemal A. Global cancer statistics 2018: GLOBOCAN estimates of incidence and mortality worldwide for 36 cancers in 185 countries. CA Cancer J Clin. 2018;68(6):394-424.

3. Siegel RL, Miller KD, Jemal A. Cancer statistics, 2018. CA Cancer J Clin. 2018: 68(1):7-30.

4. Hanahan D, Weinberg RA. Hallmarks of cancer: the next generation. Cell. 2011;144(5):646-74.

5. Yang Y. Cancer immunotherapy: harnessing the immune system to battle cancer. J Clin Invest. 2015:125(9):3335-7.

6. Bedognetti D, Ceccarelli M, Galluzzi L, Lu R, Palucka K, Samayoa J, et al. Toward a comprehensive view of cancer immune responsiveness: a synopsis from the SITC workshop. J Immunother Cancer. 2019;7(1):131.

7. Minn AJ. Interferons and the immunogenic effects of Cancer therapy. Trends Immunol. 2015;36(11):725-37.

8. Snell LM, McGaha TL, Brooks DG. Type I interferon in chronic virus infection and Cancer. Trends Immunol. 2017;38(8):542-57.
9. O'Brien TR, Prokunina-Olsson L, Donnelly RP. IFN-lambda4: the paradoxical new member of the interferon lambda family. J Interf Cytokine Res. 2014; 34(11):829-38.

10. Prokunina-Olsson L, Muchmore B, Tang W, Pfeiffer RM, Park H, Dickensheets $\mathrm{H}$, et al. A variant upstream of IFNL3 (IL28B) creating a new interferon gene IFNL4 is associated with impaired clearance of hepatitis C virus. Nat Genet. 2013;45(2):164-71.

11. Key FM, Peter B, Dennis MY, Huerta-Sanchez E, Tang W, Prokunina-Olsson L, et al. Selection on a variant associated with improved viral clearance drives local, adaptive pseudogenization of interferon lambda 4 (IFNL4). PLoS Genet. 2014;10(10):e1004681.

12. Prokunina-Olsson L. Genetics of the human interferon lambda region. J Interf Cytokine Res. 2019;39(10):599-608.

13. Hayes CN, Imamura M, Aikata H, Chayama K. Genetics of IL28B and HCV-response to infection and treatment. Nat Rev Gastroenterol Hepatol. 2012;9(7):406-17.

14. Machmach K, Abad-Molina C, Romero-Sanchez MC, Dominguez-Molina B, Moyano M, Rodriguez MM, et al. IFNL4 ss469415590 polymorphism is associated with unfavourable clinical and immunological status in HIVinfected individuals. Clin Microbiol Infect. 2015;21(3):289 e1-4.

15. Real LM, Herrero R, Rivero-Juarez A, Camacho A, Macias J, Vic S, et al. IFNL4 rs368234815 polymorphism is associated with innate resistance to HIV-1 infection. AIDS. 2015;29(14):1895-7.

16. Jaimes-Bernal C, Rallon N, Benito JM, Omar M, Gomez-Vidal MA, Marquez FJ, et al. A knockout IFNL4 variant is associated with protection from sexually transmitted HIV-1 infection. J Infect Dis. 2019;219(5):772-6.

17. Manuel O, Wojtowicz A, Bibert S, Mueller NJ, van Delden C, Hirsch $\mathrm{HH}$, et al Influence of IFNL3/4 polymorphisms on the incidence of cytomegalovirus infection after solid-organ transplantation. J Infect Dis. 2015;211(6):906-14.

18. Bibert S, Wojtowicz A, Taffe P, Manuel O, Bernasconi E, Furrer H, et al. The IFNL3/4 DeltaG variant increases susceptibility to cytomegalovirus retinitis among HIV-infected patients. AIDS. 2014;28(13):1885-9.

19. Liu J, Lichtenberg T, Hoadley KA, Poisson LM, Lazar AJ, Cherniack AD, et al. An integrated TCGA pan-Cancer clinical data resource to drive high-quality survival outcome analytics. Cell. 2018;173(2):400-16 e11.

20. R, Core, Team. R: A language and environment for statistical computing. R Foundation for Statistical Computing, Vienna, Austria URL https://www.Rprojectorg/. 2018

21. Minas TZ, Tang W, Smith CJ, Onabajo OO, Obajemu A, Dorsey TH, et al. IFNL4-DeltaG is associated with prostate cancer among men at increased risk of sexually transmitted infections. Commun Biol. 2018;1:191.

22. Tang W, Wallace TA, Yi M, Magi-Galluzzi C, Dorsey TH, Onabajo OO, et al. IFNL4-DeltaG allele is associated with an interferon signature in tumors and survival of African-American men with prostate Cancer. Clin Cancer Res. 2018:24(21):5471-81

23. Bibert $\mathrm{S}$, Wojtowicz A, Taffe $\mathrm{P}$, Tarr PE, Bernasconi E, Furrer $\mathrm{H}$, et al. Interferon lambda 3/4 polymorphisms are associated with AIDS-related Kaposi's sarcoma. AIDS. 2018;32(18):2759-65.

24. Stiff A, Carson W III. Investigations of interferon-lambda for the treatment of cancer. J Innate Immun. 2015;7(3):243-50.

25. Lasfar A, Zloza A, Cohen-Solal KA. IFN-lambda therapy: current status and future perspectives. Drug Discov Today. 2016;21(1):167-71.

\section{Publisher's Note}

Springer Nature remains neutral with regard to jurisdictional claims in published maps and institutional affiliations.

Ready to submit your research? Choose BMC and benefit from

- fast, convenient online submission

- thorough peer review by experienced researchers in your field

- rapid publication on acceptance

- support for research data, including large and complex data types

- gold Open Access which fosters wider collaboration and increased citations

- maximum visibility for your research: over $100 \mathrm{M}$ website views per year

At BMC, research is always in progress.

Learn more biomedcentral.com/submissions 